\title{
Slowly Pulsating B Stars
}

\author{
C. Waelkens \\ Instituut voor Sterrenkunde KU Leuven, Belgium
}

\begin{abstract}
We discuss two new slowly pulsating $B$ stars for which it can be convincingly shown that they are multiperiodic variables. The group now contains ten confirmed and likely multiperiodic mid-B stars with periods between 0.7 and 4.4 days. The multiperiodicity and the length of the periods point to g-mode pulsations. The very high order of the modes implies that the modes are excited in the core or in the very outer layers of the star. A recently proposed mechanism, in which both the inner and outer layers intervene, is discussed.
\end{abstract}

\section{Introduction}

The slowly pulsating B stars were introduced as a group of B-type variable stars by Waelkens and Rufener (1985) and by Waelkens (1992). They are characterized by spectral types in the range B3-B8 (masses between 3 and 6 solar masses), multiple periods of the order of days, and photometric amplitudes which increase with decreasing wavelength. Probably the line-profile variable stars 53 Persei and $\iota$ Herculis also belong to the class. However, line-profile variabilty is rather common among B-type stars, and is not always linked to periodic photometric variability; it may thus not be sufficient for the definition of a class of variables. It is for this reason that we prefer to speak about "slowly pulsating B stars" rather than "53 Persei stars".

\section{Multiperiodicity of the new slowly pulsating B stars}

The periods observed for the slowly pulsating B stars are of the order of twenty times that of the fundamental radial mode. If only one period were observed in these stars, the case for pulsation would appear fairly unconvincing; rotation would be a more promising explanation for the variations. Observations gathered over several years, however, clearly indicate multiple periods in all well observed stars.

Data gathered during a single observing season most often do not offer sufficient proof for multiperiodicity, because only a few beat periods or even a fraction of one can be observed during a single season. We have now found two examples for which the frequency spacing is large enough so as to enable us to distinguish different frequencies in the data of individual years. The clearest case is HD 45284 , for which three well resolved frequencies with similar power are found; the periods amount to 0.664 days, 0.807 days, and 0.887 days, respectively. A somewhat more complex star 
is $\mathrm{HD} 34798$ in which at least four periods in the range between 1.02 and 1.48 days occur. Both stars will be discussed extensively in a forthcoming paper.

\section{Discussion of the excitation mechanism}

High-order g-modes in B-type stars attain appreciable amplitudes only in the core and in the very outer layers; in the intermediate layers the displacement is very small. The cause of the pulsation of these stars must then reside in the deep interior or the outmost layers, or in both. The outer layers contain only a small amount of mass, so that oscillations that are only excited there would be damped in the core.

Degryse et al. (1992) have carried out a vibrational stability analysis of an evolved five-solar-mass model, and found overstability, due to the combined effects of the $\epsilon$ mechanism in the core and the HeII partial ionization in the outer layers. Some contribution to the excitation was also found in the $\mu$-gradient zone, which may prove spurious if a better treatment of semi-convection is applied. Nevertheless, it is promising that actual destabilization is found in the layers were the oscillations in these stars attain there largest amplitudes. It is also found by Degryse et al. (1992) that the modes are less unstable (though not stable) for smaller radial orders. The instability occurs only for a limited range of models, with masses near five solar mass and near the end of core-hydrogen burning, in reasonable agreement with observations.

Earlier attempts to detect overstability in B-type stars from the $\epsilon$-mechanism (e.g. Osaki 1976) all have failed. We note that these attempts were directed at understanding the $\beta$ Cephei stars, which imply more massive models and modes of lower radial order. In such hot stars the HeII partial ionization zone contributes less to the excitation of $g$-modes. Moreover, low-order modes have appreciable amplitudes throughout the star, and so also in the zones where damping is important. It is the particular aspect of high-order modes to be practically confined to a few layers in the star which may allow excitation of these modes in our stars.

The main weakness of the analysis by Degryse et al. may reside in the treatment of the $\mu$-gradient zone and semiconvection. To our knowledge a stability analysis of high-order g-modes in B-type stars has never been performed before. We would welcome an independent analysis, with a more sophisticated code, to check the results by Degryse et al.

\section{References:}

Degryse, K., Noels, A., Gabriel, M., Waelkens, C., Smeyers, P., 1992, Astron. Astrophys., in press.

Osaki, Y., 1976, Publ. Astron. Soc. Japan 28, 105.

Waelkens, C., 1992, Astron. Astrophys. 246, 453.

Waelkens, C., Rufener, F., 1985, Astron. Astrophys. 152, 6. 